\title{
Foveal Visual Strategy during Self-Motion Is Independent of Spatial Attention
}

\author{
Min Wei and Dora E. Angelaki \\ Department of Neurobiology, Washington University School of Medicine, St. Louis, Missouri 63110
}

Translational self-motion disturbs the stability of retinal images by inducing a pattern of retinal optic flow that cannot be compensated globally by a single eye movement. The eyes must rotate by different amounts, depending on which spatial location needs to be stabilized on the retina. However, compensatory eye movements during steady fixation are always such as to maintain visual acuity on the fovea at the expense of significant image slip on the peripheral retina. We investigated whether such a foveal visual strategy during translation is hard-wired or whether it embeds enough flexibility to also allow for behaviorally relevant objects outside the foveae to be stabilized preferentially on the retinas. Monkeys were moved forward or backward and leftward or rightward passively in darkness while planning a saccade or bar release to peripheral dimmed targets. By comparing the eye movements made during these tasks with those under conditions of steady fixation, we found that the motion-induced eye movements depended only on current fixation. This was true even during the last milliseconds just before a saccade to the peripheral target. We conclude that the foveal stabilization strategy is invariant and solely dependent on current eye position, a strategy that is optimal for both processing speed and efficiency in the extraction of heading information from retinal flow during self-motion.

Key words: eye movement; vestibulo-ocular reflex; spatial attention; self-motion; vergence angle; Foveal strategy

\section{Introduction}

When a moving subject experiences lateral motion, as when we look through the window of a moving car, the speed of the retinal optic flow varies with object distance. Without any compensatory eye movement, only far-fixated targets remain stable on the retina. However, when the retinal images of nearby objects must be stabilized, an eye movement is called for, the amplitude of which increases with proximity to the subject (Fig. $1 A$ ). A similar but even more complex visual challenge arises when we look into the direction of heading, as often occurs when we walk or drive a car. In this case we experience an expanding visual scene in which both the retinal speed and motion direction of nearby objects depend on their spatial location with respect to the direction of heading (Fig. $1 B$ ). Thus for images of near targets to remain stable on the retina, the amplitude and direction of compensatory eye movements must scale as a function of viewing distance and object eccentricity.

Indeed, it is now well established that moving subjects generate spatially selective, visually driven, or vestibularly driven compensatory eye movements during translation, the latter known as translational vestibulo-ocular reflex (TVOR) (Schwarz et al., 1989; Paige and Tomko, 1991; Schwarz and Miles, 1991; Busettini et al., 1997; Yang et al., 1999; Kodaka et al., 2003). However,

Received Sept. 20, 2005; revised Nov. 24, 2005; accepted Nov. 25, 2005.

This work was supported by National Institutes of Health Grants R01 EY12814 and R01 EY15271.

Correspondence should be addressed to Dr. Dora E. Angelaki, Department of Anatomy and Neurobiology, Box 8108, Washington University School of Medicine, 660 South Euclid Avenue, St. Louis, M0 63110. E-mail: angelaki@pcg.wustl.edu.

DOI:10.1523/JNEUROSCI.3986-05.2006

Copyright $\odot 2006$ Society for Neuroscience $\quad$ 0270-6474/06/260564-09\$15.00/0 unlike the more traditional VOR elicited during rotational motion, which is relatively independent of target location and for which the goal is to stabilize images on the entire retina, no single eye movement generally could result in stabilization of the whole visual field during translation. Given the high importance of the fovea in primate vision, it is not surprising that the TVOR stabilizes images on the fovea, thus allowing image blur in the retinal periphery (Paige and Tomko, 1991; McHenry and Angelaki, 2000; Angelaki and Hess, 2001; Hess and Angelaki, 2003).

However, all such studies showing that humans and monkeys use a foveal visual strategy during self-motion have used conditions of steady fixation in which the locus of spatial attention and the position of the eyes always coincide. Nonetheless, it might be functionally appropriate for these eye movements to have a flexible spatial goal, such that objects at other (e.g., peripheral) retinal locations instead can be stabilized preferentially whenever it is behaviorally relevant. This hypothesis has been investigated here by the study of motion-driven compensatory eye movements while a saccade or bar release is planned in response to the dimming of a peripheral target (Goldberg and Wurtz, 1972; Wurtz and Mohler, 1976). If the vestibulo-motor goal is always to keep images stable on the foveae, eye movements always should be determined by the location of the fixated target (foveal visual strategy). Alternatively, for a flexible sensorimotor goal, behaviorally relevant peripheral target locations (i.e., objects that do not fall on the two foveae) could be stabilized preferentially instead. In this case the eye movements elicited in the saccade/bar release tasks would be similar to those during steady fixation of the peripherally located target (flexible goal visual strategy). We present evidence for a preferential and hard-wired stabilization of 


\section{A}

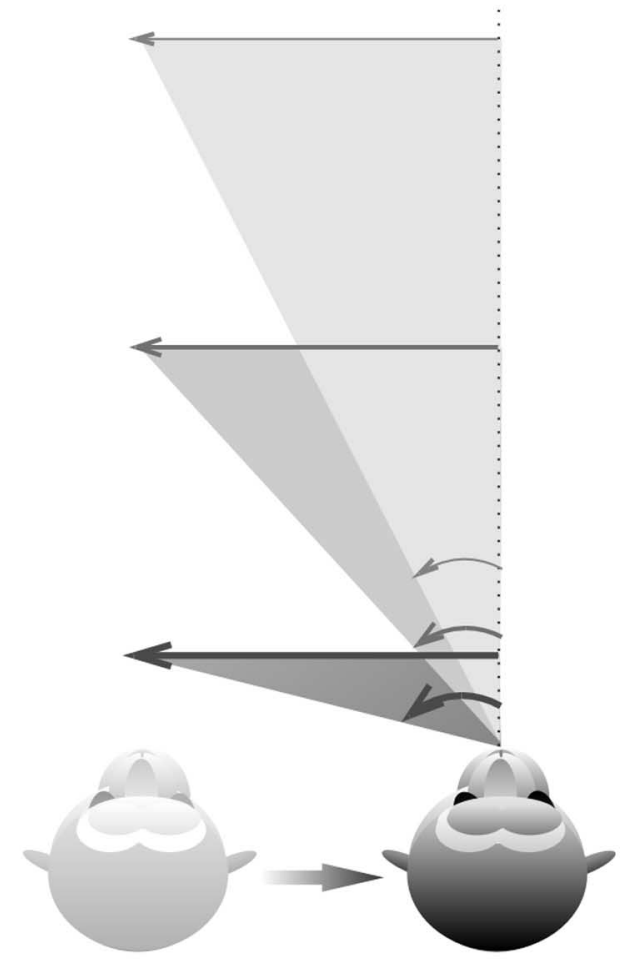

B

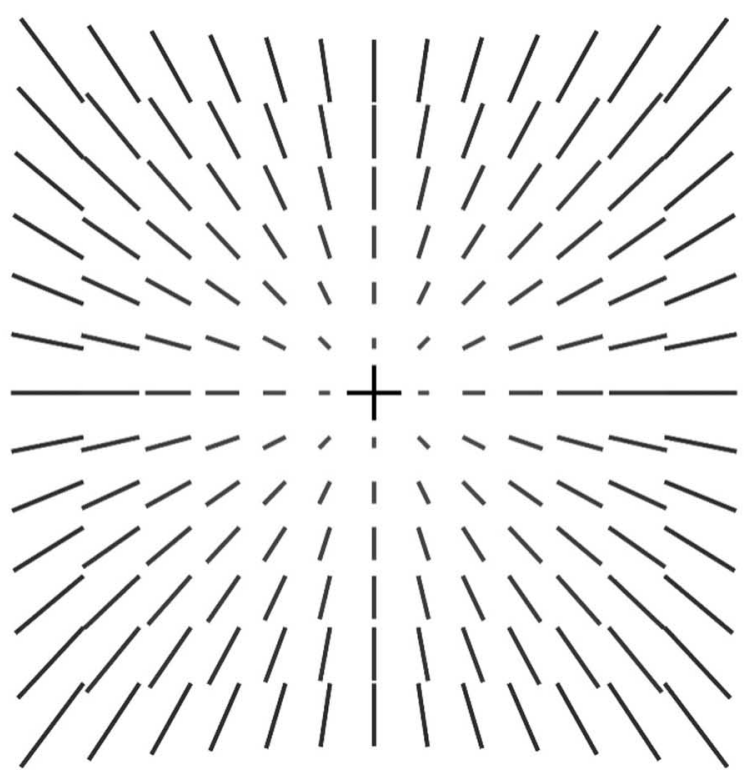

Figure 1. Schematic diagrams of the retinal optic flow experienced by a moving subject during linear self-motion. $\boldsymbol{A}$, During translation to the right (gray-shaded arrow), the retinal images slip on the retina in the opposite direction (left), requiring a compensatory eye movement that varies inversely proportionally to target distance. Thus, the closer the target is, the larger the eye movement necessary to keep its image stable on the retina. $\boldsymbol{B}$, During forward translation, the subject experiences an expanding retinal flow in which both the amplitude and direction of experienced optic flow depend on the eccentricity of the object with respect to the direction of heading. images on the fovea, even immediately before a saccade to the peripheral target.

\section{Materials and Methods}

Subjects and experimental setup. Two rhesus monkeys were chronically implanted with a circular, lightweight Delrin head restraint ring anchored to the skull with stainless steel screws and T-bolts (for details, see Angelaki, 1998). Binocular horizontal and vertical eye movements were recorded by using scleral eye coils surgically implanted under the conjunctiva of each eye (Robinson, 1963; Judge et al., 1980). All surgical procedures and animal handling were in accordance with institutional and National Institutes of Health guidelines.

During experiments each head-restrained animal was seated upright in a primate chair secured inside the inner frame of a vestibular turntable consisting of a three-dimensional rotator on top of a $2 \mathrm{~m}$ linear sled (Acutronics, Pittsburgh, PA). The linear acceleration of the head was measured with a three-axis accelerometer (Crossbow Technology, San Jose, CA) mounted on the head ring support structure. Binocular horizontal and vertical eye movements were measured by the use of a threefield magnetic coil system (CNC Engineering, Seattle, WA). Eye movement signals were low-pass-filtered with six-pole Bessel filters at $200 \mathrm{~Hz}$. The analog signals were digitized at $833.3 \mathrm{~Hz}$ with 16-bit resolution and stored for off-line analysis. Finally, a custom-written script in Spike2 software controlled the stimulus presentation, the motion of the sled, and data acquisition hardware (CED Power 1401; Cambridge Electronic Design, Cambridge, UK).

Peripheral visual targets for the saccade/bar release tasks consisted of eight red light-emitting diodes (LEDs) mounted on an earth-horizontal plane at two distances $(12$ and $100 \mathrm{~cm})$ from the animal. Each of the four targets at each viewing distance was placed 10 and $20^{\circ}$ to the right and left from the animal's midsagittal plane. Two additional dual-color (red/ green) central LEDs at distances of 12 and $57 \mathrm{~cm}$ served as the central fixation targets.

Training. Animals were trained to perform two simple tasks. In the bar release task the animal was trained to press and hold down a bar, at which time one of the central fixation targets (red) was turned on in a softly illuminated laboratory room (Fig. 2A, panel 1) (Goldberg and Wurtz, 1972; Wurtz and Mohler, 1976). After the animal fixated on the central target for $\sim 1 \mathrm{~s}$, one of the peripheral targets was turned on for a random period of $\sim 50-3000 \mathrm{~ms}$ and then suddenly was dimmed by reducing its power by $\sim 50 \%$ for $600-800 \mathrm{~ms}$ (Fig. $2 A$, panels 2,3 , respectively). The animal was rewarded with drops of juice if he released the bar within $150-600 \mathrm{~ms}$ after the peripheral target was dimmed (Fig. $2 \mathrm{~A}$, panel $5 \mathrm{a}$, bar release) while continuing to fixate on the central target (with vergence and version windows of \pm 0.5 and $\pm 0.75^{\circ}$, respectively). Whenever either central target fixation was broken or the bar was released prematurely, the trial was aborted, and the data were discarded. All targets were turned off for $\sim 4$ s between trials.

The saccade task was identical to the bar release task described above, except that the central fixation target was green and the animal was instructed to make a saccade to the peripheral target, rather than to release the bar, with its dimming (Fig. $2 \mathrm{~A}$, panel $5 \mathrm{~b}$, saccade). Animals had to make a saccade within $100-500 \mathrm{~ms}$ of peripheral target dimming, with their eyes landing within $\pm 0.5^{\circ}$ (vergence) and $\pm 0.75^{\circ}$ (version) of the target to get a juice reward. We used both the bar release and saccade tasks such that spatial attention would be dissociated from the fixation target without or with an associated saccade. In particular, unlike the bar release task in which there were no saccadic premotor commands, the saccadic task tested TVOR flexibility during the development of oculomotor commands.

Experimental protocols. First the animals were trained extensively with the saccade/bar release tasks while they remained stationary. The monkeys also were trained to hold vergence angle stable (within $\pm 1^{\circ}$ ) for a few hundred milliseconds in complete darkness (Wei et al., 2003; Wei and Angelaki, 2004). Subsequently, the actual experimental protocol consisted of either lateral (left or right) or fore-aft passive head and body motions (Fig. 2A, panel 4). The motion profile consisted of a position ramp $( \pm 5 \mathrm{~cm})$ with peak acceleration of $196 \mathrm{~cm} / \mathrm{s}^{2}(0.2 \mathrm{~g})$. The shortduration $(500 \mathrm{~ms})$ motion occurred during the reaction time at several 
A

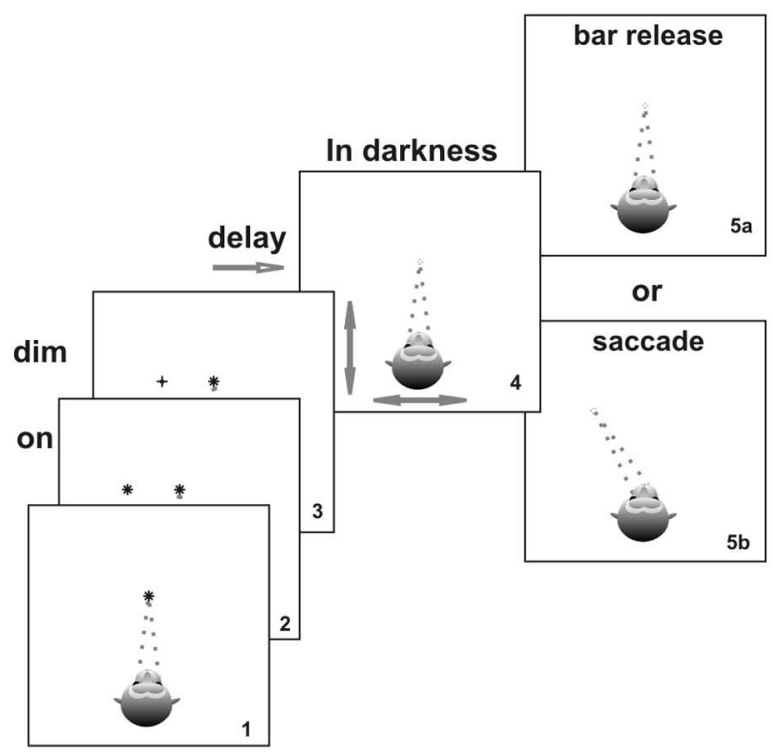

B

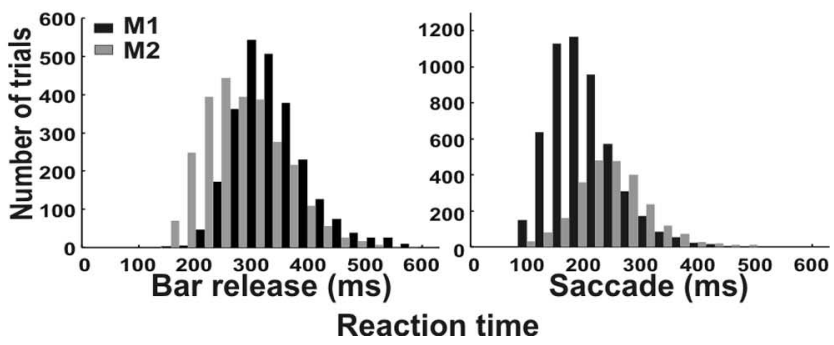

Figure 2. Behavioral task design and parameters. $A$, Schematic of experimental protocol for the saccade/bar release tasks. After fixation of a central target (1), a peripherally located LED first was illuminated (2) and subsequently ( $50-3000 \mathrm{~ms}$ later) was dimmed (3). At different delays after peripheral target dimming and during continued fixation on the central target, the animal was moved passively leftward/rightward or forward/backward (4, arrows) in darkness. The animals were trained either to release a pressed bar (5a) or to make a saccade $(5 b)$ to the peripheral target location as soon as it was dimmed. $\boldsymbol{B}$, Reaction time distributions for bar release (left) and saccade onset (right), plotted separately for the two animals M1 (black) and M2 (gray).

different delays after target dimming (bar release task, 50-300 ms; saccade task, 25-150 ms), with all motion delays randomly interleaved within each experimental session. Immediately before the sled movement onset all LEDs and room lights were turned off to keep the animal in complete darkness during motion (to elicit the TVOR). Data were collected after $\sim 1$ week of familiarizing the animals to the motion profiles (with the animals' performance at an $80-90 \%$ success rate).

For the lateral motion tasks the $57 \mathrm{~cm}$ central LED served as the fixation target, and all eight eccentric LEDs (both near and far) were used as the peripheral targets. For the fore-aft motion tasks the $12 \mathrm{~cm}$ central LED served as the fixation target, with the four near $(12 \mathrm{~cm})$ eccentric LEDs being used as the peripheral targets. The fixation targets were different for lateral and forward/backward motion because of the relatively small amplitude of the fore-aft TVOR and the fact that robust compensatory eye movements are elicited only during the fixation of near targets. For this reason also, only the near $(12 \mathrm{~cm})$ LEDs were used as peripheral targets during the fore-aft motion tasks.

The motion runs always were interleaved randomly with stationary runs within the same block of trials. Although there was no behavioral window during motion (thus allowing the animal to generate TVOR in the dark), trained animals were required to maintain central target fixation (vergence, $\pm 1^{\circ}$; version, $\pm 1.5^{\circ}$ ) during the first $300 \mathrm{~ms}$ in darkness during the corresponding stationary trials. Because the randomly interleaved motion and stationary trials were identical in all other respects, animals were motivated to maintain appropriate binocular fixation on the central target for short periods in darkness without the reinforcement of any behavioral window during motion. Only motion trials have been included in the off-line analyses.

In addition to these saccade/bar release motion tasks, fixation control experiments in which animals were moved in darkness during the fixation of each of the central and peripheral LEDs were performed also. The motion direction was leftward, rightward, forward, or backward and had an identical temporal profile to that used for the saccade/bar release tasks. Behavioral windows and all other parameters were also identical for the steady fixation controls and saccade/bar release tasks. Data were collected on multiple experimental days (a minimum of $10 \mathrm{~d}$ for each task), and all were combined for off-line analyses.

Data analysis. Raw eye movement data were analyzed off-line with custom-written scripts in MatLab (MathWorks, Natick, MA). Eye movements were calibrated first by using a daily fixation task, with positive eye positions corresponding to downward and leftward directions. Horizontal eye velocity was computed by time differentiation of eye position via a polynomial filter (Savitsky and Golay, 1964). Because of the mostly horizontal location of all LEDs, vertical eye movement changes were small (thus not included in this analysis). A custom-written script allowed the experimenter to examine each run manually by plotting the horizontal eye position and velocity of each eye as a function of time. For the bar release task the reaction times were defined as the time period between target dimming and bar release. For the saccade task the reaction times were defined as the time interval between target dimming and saccade onset. The latter was estimated by detecting the time when either conjugate or vergence eye acceleration first exceeded $900 \% \mathrm{~s}^{2}$ (a value that always exceeded the near TVOR responses of the control task). A line then was fit to the previous seven data points $(8.4 \mathrm{~ms})$. Saccade/vergence onset (abbreviated as saccade onset in subsequent analysis) was taken at the point at which this line intersected the acceleration response profile. Mean $( \pm$ SD) reaction times were $346 \pm 65 \mathrm{~ms}$ (animal M1) and $289 \pm 71$ $\mathrm{ms}$ (animal M2) for the bar release task (Fig. $2 B$, left) and $205 \pm 57 \mathrm{~ms}$ (M1) and $252 \pm 64 \mathrm{~ms}$ (M2) for the saccade task (Fig. 2 B, right).

For both the saccade/bar release and steady fixation control tasks the horizontal left and right eye position and velocity from individual runs subsequently were aligned on motion onset, as determined by the linear acceleration stimulus (rather than response onset). For lateral motion responses the analyses used the first $100 \mathrm{~ms}$ after motion onset. Because of the more transient nature of fore-aft TVOR responses (Hess and Angelaki, 2003), data were analyzed only during the first $55 \mathrm{~ms}$ of motion. We then computed mean responses for the control task separately for left/right eye, motion direction, and LED location to use as a reference for comparison with the eye movements elicited during the saccade/bar release tasks (only saccade-free responses were used for computing these control averages).

Data during the saccade/bar release tasks were processed two different ways, grouped according to either motion onset delay or saccade/bar release reaction time. For the first analysis the horizontal left and right eye position and velocity from individual runs during the saccade/bar release tasks were averaged separately for each motion direction, peripheral LED location, and motion onset delay. Because the latter was a continuous variable, delay times within $\pm 10 \mathrm{~ms}$ of $50,100,150,200,250,300 \mathrm{~ms}$ (bar release task) or $25,50,75,100,125,150 \mathrm{~ms}$ (saccade task) were pooled together in the analyses. To exclude trials in which the detected bar release/saccade could be cued by the motion onset itself rather than by the previous dimming of the peripheral target, we analyzed only trials in which bar release and saccade reaction times relative to the onset of the motion stimulus were $<250$ and $175 \mathrm{~ms}$, respectively (Bowman et al., 1993; Kustov and Robinson, 1996; Reynolds et al., 2000; Cook and Maunsell, 2004; Li et al., 2005) (corresponding to $81 \%$ of the data from animal 1 and $70 \%$ of the data in animal 2).

To test the predictions of the foveal and flexible goal hypotheses, we computed two metrics, a flexible goal index, FlexI, and a foveal index, FovI, as follows. The FlexI metric was defined as the difference in mean horizontal eye velocity (taken at different times after motion onset) be- 
tween the saccade/bar release task $(A)$ and central target fixation control task $(C C)$, normalized by the absolute value of the latter as follows: FlexI $=(A-C C) /|C C|$. The FovI metric was computed as the normalized difference in mean horizontal eye velocity between the peripheral $(C P)$ and $C C$ tasks as follows: FovI $=(C P-C C) /|C C|$. When FlexI was plotted versus the corresponding FovI, the flexible goal visual strategy hypothesis expects data to fall along the unity slope diagonal line. In contrast, the foveal visual strategy hypothesis predicts data points falling along the abscissa. Relationships between these metrics were quantified with linear regression, obtained by minimizing the perpendicular offset of the data to the line (using a nonlinear least-squares algorithm based on the interior-reflective Newton method) (Coleman and Li, 1996). The latter analysis was used for independent variables with $95 \%$ confidence intervals computed by using bootstrapping with replacement.

The previous analysis used averaging based on the motion onset delay relative to target dimming without any consideration for the bar release or saccade reaction times. Thus to verify that the foveal visual strategy prevailed throughout the last tens of milliseconds before saccade or bar release onset, we also used mean responses during the steady fixation control task to compute a normalized central-to-peripheral transition time-dependent index (CPT) for the lateral motion tasks. For each saccade/bar release run, $A(t)$, CPT was computed as follows: $\mathrm{CPT}(t)=$ $(A(t)-C C(t)) /(C P(t)-C C(t))$, where $C C(t)$ and $C P(t)$ are the mean responses for the central and peripheral targets during the steady fixation control task. Note that, similar to the previous analysis, we first synchronized all of the data with respect to motion onset and then calculated the $C P(t)$ and $C C(t)$ averages before computing the CPT $(t)$ index for each $A(t)$ trial. Because TVOR is functionally important within the first hundred millisecond of motion (Schwarz et al., 1989) and because of TVOR latency (10-20 ms) (Bush and Miles, 1996; Angelaki and McHenry, 1999), only data within 30-100 ms of lateral motion onset were included in this computation.

Once such a normalized time-dependent index was computed for each trial, it was time-shifted to align the normalized response relative to the bar release or saccade onset times. Normalized responses were averaged separately for each motion direction and plotted as a function of time relative to bar release or saccade onset. Note that this averaging based on saccade/bar release onset was possible because responses were independent of motion onset delay relative to target dimming (see Fig. 5). The $\mathrm{CPT}$ index was calculated by using the eye response with the largest differences between central and peripheral fixation, i.e., from right eye responses during leftward motion and left eye responses during rightward motion. The foveal visual strategy hypothesis predicts $\mathrm{CPT} \sim 0$, whereas the flexible goal hypothesis predicts $\mathrm{CPT} \sim 1$. Note that, although the definition of this index is similar to the FlexI versus FovI metric slopes, the fact that the CPT index is computed according to the reaction time for each trial allows for a direct quantification of how similar the saccade/bar release task responses are to those of the central versus peripheral target fixation control tasks just before the bar release or saccadic eye movement.

\section{Results}

Two rhesus monkeys were trained to either release a bar or make a saccadic eye movement within a few hundred milliseconds after a peripheral target was dimmed while they fixated on a centrally located target (Fig. 2A). At unpredictable intervals after the peripheral target was dimmed, all targets were turned off and the animal was moved passively leftward/rightward or forward/backward. Depending on the horizontal eye movement amplitude, only images at either central or peripheral spatial locations can remain stable during motion. If the vestibulo-motor goal is always to keep images stable on the foveae, the eye movements elicited during the saccade/bar release tasks would be indistinguishable from those elicited during steady fixation of the central target (foveal visual strategy). Alternatively, the goal of the vestibulo-ocular system could be flexible, such that peripheral target locations (i.e., objects that do not fall on the two foveae) also could be stabilized preferentially when image stability at these locations becomes of behavioral relevance. In the latter case the eye movements elicited in the saccade/bar release tasks might be similar to those during steady fixation of the peripherally located (rather than the central fixation) targets (flexible goal visual strategy).

With these predictions in mind, we next describe the motioninduced eye movements during the steady fixation and saccade/ bar release tasks. Lateral displacements result in planar retinal flow fields that require a compensatory eye movement that varies more with viewing distance than with target eccentricity (at least in the $\pm 20^{\circ}$ range) (Fig. $1 A$ ). In contrast, forward/backward displacements result in a radial flow pattern that requires compensatory eye movements for which the amplitude and direction depend strongly on object eccentricity with respect to the direction of heading (Fig. $1 B$ ). Because of these differences for lateral versus fore-aft motion, extrafoveal object location relative to the fixation target was varied in depth during lateral motion and in eccentricity during fore-aft motion.

\section{Lateral motion tasks}

The effects of target distance on the eye movements elicited during rightward motion under conditions of steady fixation are illustrated with an example from animal M1 in Figure $3 A$. The top traces (far target) illustrate left and right eye position and velocity, superimposed from several trials, for a relatively far target $(57 \mathrm{~cm})$. The bottom traces (near target) show the response to the same motion when the fixation target is near $(12 \mathrm{~cm})$. Consistent with previous studies (Schwarz et al., 1989; Paige and Tomko, 1991), horizontal eye velocity responses were small for far targets and progressively larger for near fixations. Furthermore, the closer the target was, the larger the difference in the velocity of the two eyes, an experimental observation that is consistent with the geometry characterizing an eye rotation that compensates for head translation (Angelaki and McHenry, 1999; Angelaki, 2002).

The difference in eye velocity as a function of fixation target distance, illustrated in Figure $3 A$, provides a clear distinction between the predictions of the foveal and flexible goal visual strategy hypotheses. Specifically, when a subject fixates on the far (57 $\mathrm{cm})$ target but depends on the near $(12 \mathrm{~cm})$ target for the subsequent saccade/bar release, is the reflexive eye movement that is elicited appropriate to stabilize one or the other target on the retina? The answer to this question is illustrated with the data in Figure $3 \mathrm{~B}$. Clearly, the eye velocity generated under these conditions is more similar to that during steady fixation of the far rather than the near target.

Mean \pm SE left and right eye velocity during the steady fixation and saccade/bar release tasks for the same targets as in Figure 3 are illustrated in Figure $4 A-C$. Two additional observations can be made from these plots. First, the fact that the elicited eye velocity followed the current foveal goal was independent of the relative timing between target dimming and motion onset. Second, responses were similar when the animals reported the time of dimming with a bar release (Fig. $4 B$ ) or with a saccadic eye movement (Fig. 4C). Thus, even in situations in which the dimmed target is the goal of a saccade that would place the foveae on the peripheral spatial location (i.e., in the presence of developing saccadic motor commands), the reflexive eye velocity generated before the saccadic eye movement appeared dependent on the current foveal goal, i.e., its amplitude remained appropriate to stabilize the central fixation target. Qualitative similar obser- 


\section{A Fixation task}

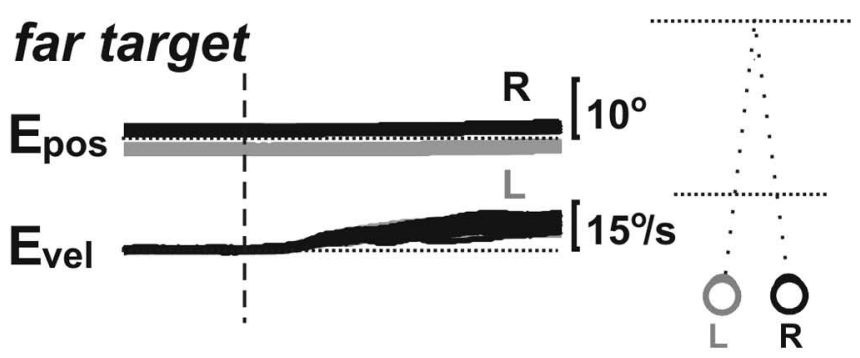

near target

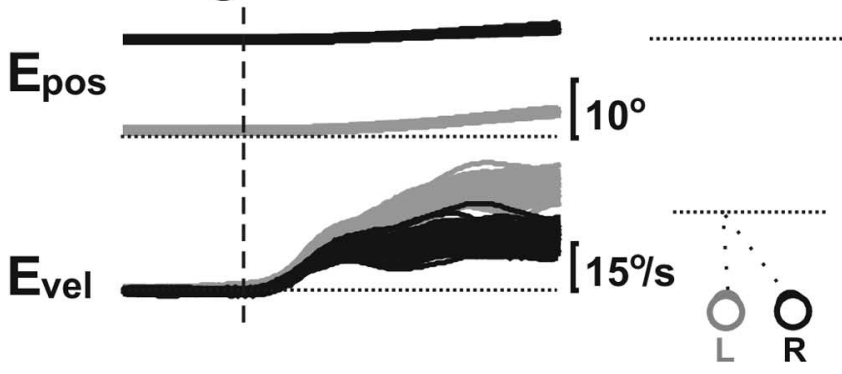

B Bar release task
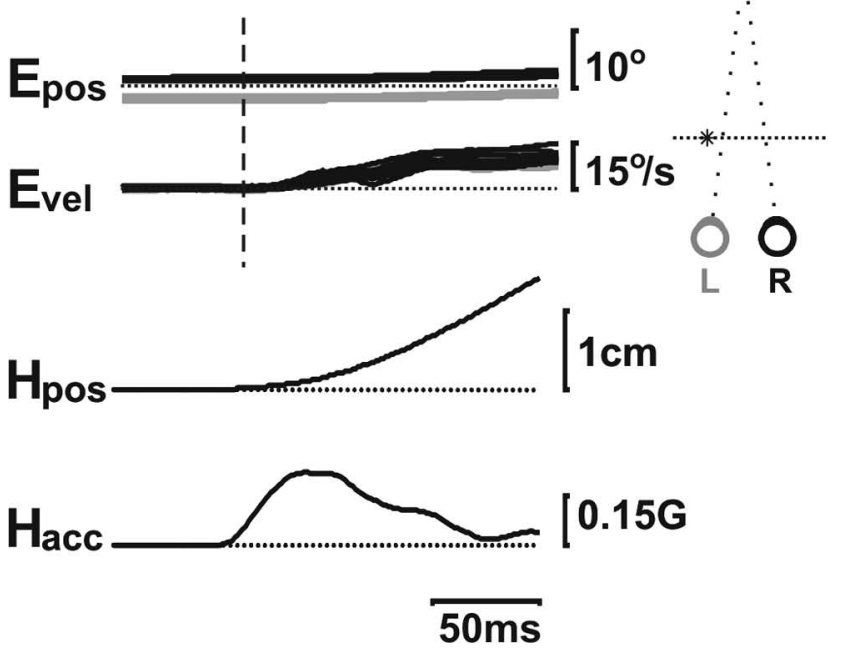

Figure 3. Lateral motion tasks. Superimposed left eye ( $L$, gray) and right eye ( $R$, black) position and velocity responses, aligned at motion onset (vertical dashed lines), are shown. $\boldsymbol{A}$, Data ( $n=36$ for near and $n=42$ for far target) during steady fixation of a central target at 57 $\mathrm{cm}$ (top) or a peripheral ( $10^{\circ}$ left) target at $12 \mathrm{~cm}$ (bottom). Eye velocity depends on target distance, with left eye responses being larger than those of the right eye during motion to the right and the reverse being true during motion to the left (Angelaki, 2002). B, Data $(n=14)$ during the bar release motion task (delay, $100 \mathrm{~ms}$ ), with the central fixation target at $57 \mathrm{~cm}$ (same as in $\boldsymbol{A}$, top) and the peripheral dimmed target at $12 \mathrm{~cm}$ (same as in $\boldsymbol{A}$, bottom). Dotted lines illustrate zero position and velocity. The bottom traces in $\boldsymbol{B}$ illustrate the mean sled position $\left(H_{\text {pos }}\right)$ and linear acceleration $\left(H_{\text {acc }}\right)$ stimulus profiles. Data are from $\mathrm{M} 1$ during translation to the right (eliciting leftward eye movements).

vations to those described for the near $(12 \mathrm{~cm})$ also held true for the far $(100 \mathrm{~cm})$ peripheral targets.

These results from the saccade/bar release task with a motion onset delay of $100 \mathrm{~ms}$ have been summarized for both animals, both motion directions, and all eight near $(12 \mathrm{~cm})$ and far $(100$ $\mathrm{cm})$ targets in Figure $5 \mathrm{~A}$. For a comparison between the predic-

\section{A Fixation task}

\section{far target \\ near target}

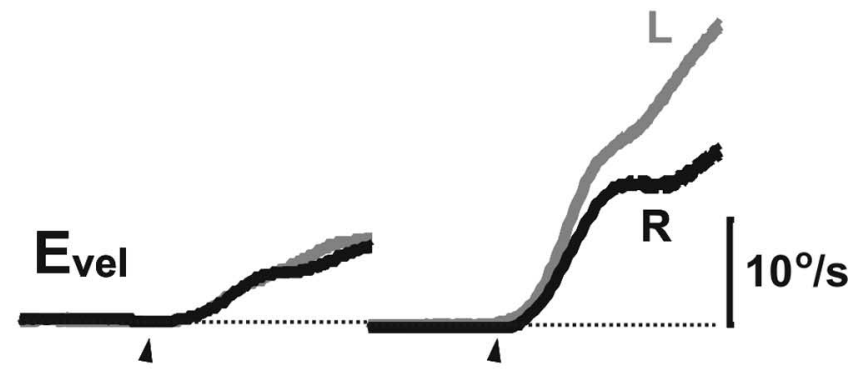

B Bar release task

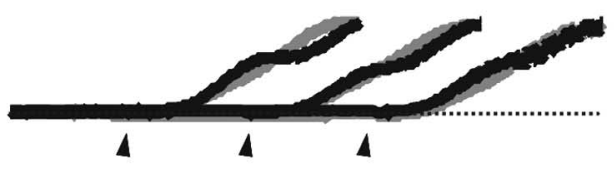

$10 \% \mathrm{~s}$

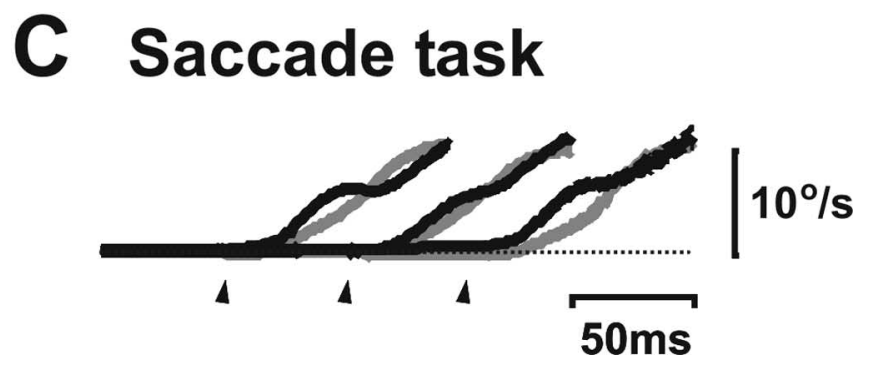

Figure 4. Lateral motion tasks. The mean \pm SE left and right eye velocity, aligned at motion onset (arrows) during steady fixation of a central target at $57 \mathrm{~cm}\left(\boldsymbol{A}\right.$, left) or a peripheral $\left(10^{\circ}\right.$ left) target at $12 \mathrm{~cm}(\boldsymbol{A}$, right), and the bar release $(\boldsymbol{B})$ or saccade motion $(\boldsymbol{C})$ tasks (delays, 50 , 100 , and $150 \mathrm{~ms}$ ), with the central fixation target at $57 \mathrm{~cm}$ and the peripheral dimmed target at $12 \mathrm{~cm}$ (same as in $\boldsymbol{A}$ ), is shown. Dotted lines illustrate zero velocity. Data are from M1 during translation to the right; number of trials is $9-52$.

tions of the foveal and flexible goal strategy hypotheses, we plotted a flexible goal index, FlexI, as a function of a foveal index, FovI (see Materials and Methods). The FlexI metric is defined as the normalized difference in mean horizontal eye velocity $45 \mathrm{~ms}$ after motion onset between the bar release and central target fixation task. The FovI is the corresponding normalized difference between the peripheral and central target fixation tasks. The flexible goal visual strategy hypothesis expects the two indexes to be identical (because saccade/bar release task responses would be the same as those during fixation of the peripheral target); thus data should fall along the unity slope diagonal line (Fig. $5 \mathrm{~A}$, dashed line). In contrast, the foveal visual strategy hypothesis, in which saccade/bar release task responses should be the same as those of the central target fixation control task, predicts data points falling along the abscissa (Fig. 5A, dotted line). Data from both animals fell along the predictions of the foveal visual strategy hypothesis [slopes of -0.009 (M1) and -0.001 (M2)].

The slopes of these regressions ( $\pm 95 \%$ confidence intervals) have been summarized for both saccade/bar release tasks and animals by plotting the data as a function of motion delay in 

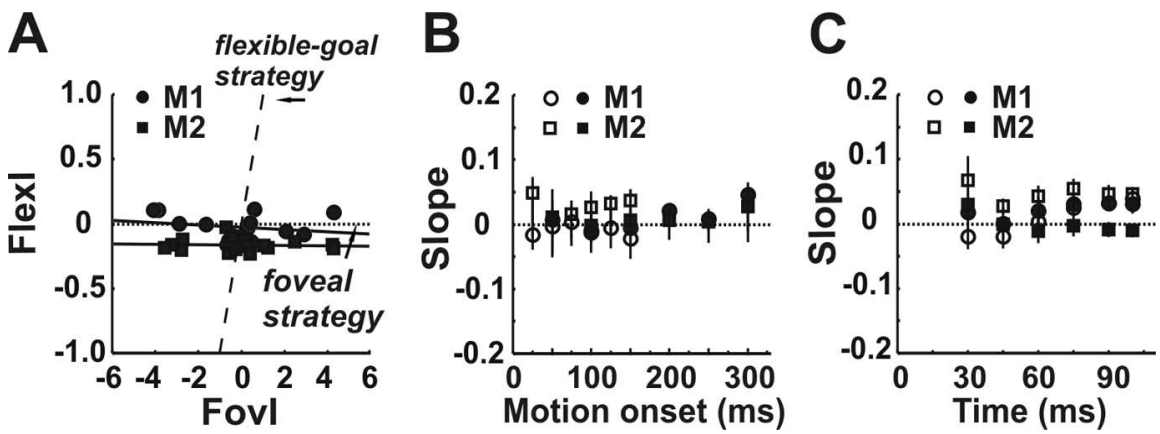

Figure 5. Summary of results for the lateral motion tasks: test of the foveal (dotted lines) and flexible goal (dashed lines) visual strategy hypotheses. A, A flexible goal index (Flexl) during the bar release task has been plotted as a function of a foveal index (Fovl; see Materials and Methods), both computed at $45 \mathrm{~ms}$ after motion onset (delay, $100 \mathrm{~ms}$ ). These values were computed separately for each peripheral LED location (8) and each motion direction (rightward/leftward). Solid lines illustrate linear regressions. $\boldsymbol{B}$, Summary of the regression slopes ( $\pm 95 \%$ confidence intervals), plotted as a function of motion onset delay (data from the right eye). $C$, Mean \pm SD slopes averaged across all motion onset delays for left/right eye data and measured at different times after motion onset ( $30-100 \mathrm{~ms}$ ). Data are plotted separately for the two animals, M1 (circles) and M2 (squares), as well as for the bar release (filled symbols) and saccade (open symbols) tasks.

\section{A Bar release task $B$ Saccade task}

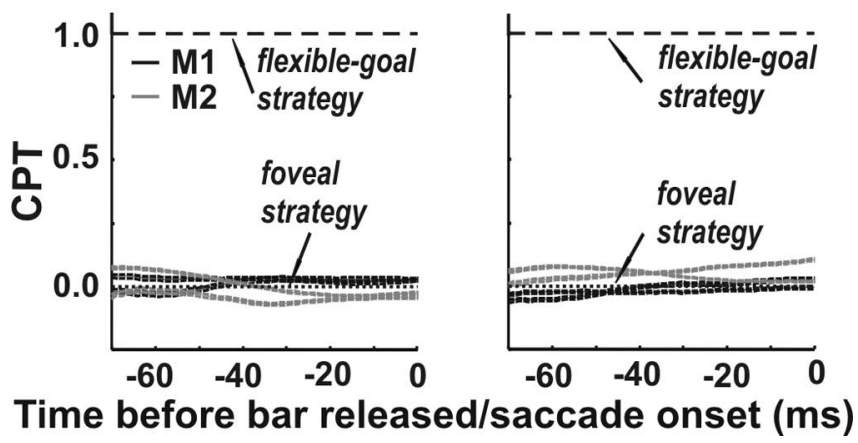

Figure 6. Normalized CPT time-dependent index during the lateral motion tasks: test of the foveal (dotted lines, (PT $\sim 0$ ) and flexible goal (dashed lines, (PT 1) visual strategy hypotheses. Because CPT was computed as a function of time before the bar release $(\boldsymbol{A})$ or saccade onset $(\boldsymbol{B})$, a direct quantification of how similar the bar release/saccade task responses were to those of the central and peripheral target fixation control tasks was possible. Data represent the means \pm SE for the near target responses, computed separately for each monkey and motion direction. Number of trials ranged between 258 and 758 .

Figure $5 B$. Regression line slopes were independent of task type (saccade/bar release) and motion delay (factorial ANOVA; $p>$ 0.05). Regression slopes averaged $( \pm \mathrm{SD})-0.009 \pm 0.02(\mathrm{M} 1)$ and $0.013 \pm 0.02(\mathrm{M} 2)$, values that were statistically different from a slope of 1 (the prediction of the flexible visual strategy hypothesis; Student's $t$ test; $p \ll 0.001$ ). When these analyses were repeated for mean eye velocity values taken at different times after motion onset, the results were similar. This is illustrated in Figure $5 C$, which plots mean \pm SD slopes computed from eye velocity $30,45,60,75,90$, and $100 \mathrm{~ms}$ after lateral motion onset separately for the bar release and saccade tasks (but averaged across all motion delays).

In addition to the analysis described above in which data were grouped according to motion onset delay, we also computed a normalized time-dependent CPT index, which is similar to the slopes described in the previous analysis. However, because the time evolution of this index was expressed relative to the bar release or saccade onset, it allowed for a direct test of the predictions from the two hypotheses during the last tens of milliseconds of the reaction time. Specifically, the foveal strategy hypothesis predicts CPT $=0$, whereas the flexible goal hypothesis expects $\mathrm{CPT}=1$. As illustrated in Figure 6, data supported the former predictions, with CPT values hovering at $\sim 0$, even during the last few milliseconds before bar release (Fig. 6A) or saccade onset (Fig. 6B). We conclude that lateral motion responses are dependent mainly on current fixation position, even before a saccadic eye movement that would bring the fovea onto the spatial location of the peripheral target.

\section{Fore-aft motion tasks}

During forward or backward motion robust reflexive eye movements are elicited only during fixation of near targets. For this reason both the central fixation and the four peripheral targets were at a distance of $12 \mathrm{~cm}$ from the animal (see Materials and Methods). Because of the radial flow pattern experienced during forward and backward motions, the eye velocity amplitude is expected to have a strong dependence on target eccentricity (Fig. $1 B$ ). For a straight-ahead target in the midsagittal plane only a small eye movement that is opposite for the two eyes (i.e., a vergence response) is necessary to stabilize its image. In contrast, targets located to the left of straight ahead require a leftward compensatory eye movement during forward motion, whereas for targets to the right the appropriate eye movement should be rightward. Consistent with previous studies (McHenry and Angelaki, 2000; Hess and Angelaki, 2003), animals generated the appropriate pattern of eye velocities, as illustrated with the examples during steady fixation of a central and an eccentric $\left(20^{\circ}\right.$ right) target in Figure $7 A$.

These large differences in eye velocity illustrated during forward motion in Figure $7 A$ provide a clear distinction between the predictions of the foveal and flexible goal visual strategy hypotheses. Specifically, when a subject fixates on the central target but depends on the eccentric target for the subsequent saccade/bar release, for which of the two targets is the reflexive eye movement appropriate? As illustrated in Figure 7, $B$ and $C$, which plots the mean $\pm \mathrm{SE}$ of left and right eye velocities during the bar release and saccade tasks for the same two targets as in Figure $7 A$, eye velocity is more similar to that during steady fixation of the central than peripheral target.

The observation that eye movements during fore-aft motion were not affected by the behavioral requirements for the saccade/ bar release tasks was independent of the relative timing between target dimming and motion onset and independent of whether the animals reported the dimming time with a bar release or a saccadic eye movement (factorial ANOVA; $p>0.05$ ). These effects were quantified by using the same metrics as those used for lateral motion. Mean \pm SD FlexI versus FovI slopes, computed from eye velocity 30,45 , and $55 \mathrm{~ms}$ after fore-aft motion onset, have been summarized separately for saccade and bar release tasks in Figure 8. Regression line slopes were statistically different from a slope of 1 (Student's $t$ test; $p \ll 0.001$ ), the prediction of the flexible goal strategy hypothesis.

\section{Discussion}

We have shown here that only the spatial location specified by the current position of the eyes is stabilized selectively on the retina 


\section{A Fixation task}

\section{central target eccentric target}
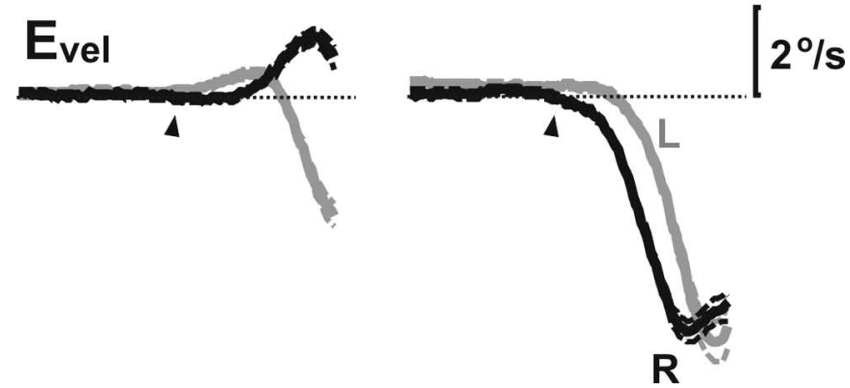

B Bar release task

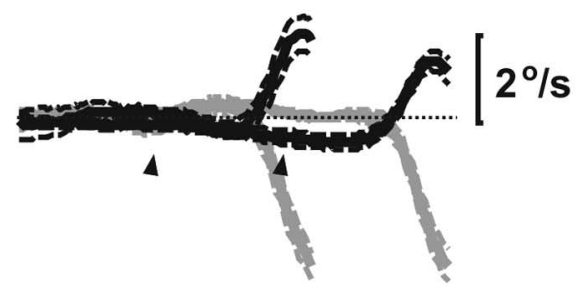

\section{Saccade task}

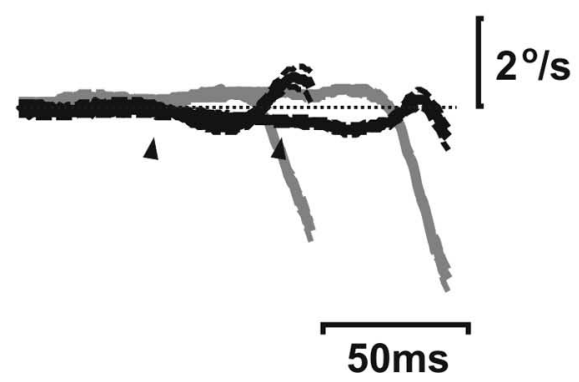

Figure 7. Forward motion task. The mean \pm SE left and right eye velocity, aligned at motion onset (arrows) during steady fixation of a central or peripheral eccentric ( $20^{\circ}$ right) target $(\boldsymbol{A})$ and the bar release $(\boldsymbol{B})$ or saccade motion $(\boldsymbol{C})$ tasks (delays, 50 and $100 \mathrm{~ms}$ ), with the central fixation and peripheral dimmed targets as in $\boldsymbol{A}$, is shown. Dotted lines illustrate zero velocity. Data are from $M 1$; number of trials is $9-42$.

during self-motion. This conclusion was reached by using simple tasks in which the functional demands for image stabilization at central (foveal) versus peripheral spatial locations were dissociated. These tasks, in which the monkey fixated on a central target but reported (via either a bar release or a saccadic eye movement) the dimming of a peripheral, eccentric target, have been used previously to allocate spatial attention (Goldberg and Wurtz, 1972; Wurtz and Mohler, 1976). Spatial attention enhances selective processing of visual information (Bashinski and Bacharach, 1980; Posner, 1980; Downing, 1988; Butter et al., 1989; Bowman et al., 1993; Carrasco et al., 2000, 2004), yet we found that the elicited eye movement was determined exclusively by the spatial location of the fixated target.

Although spatial attention was not measured directly in the present experiments, it is important to emphasize that results were identical for the bar release and saccade tasks. Because of the tight link between attention and saccadic eye movements (Kowler et al., 1995; Moore and Fallah, 2001), spatial attention

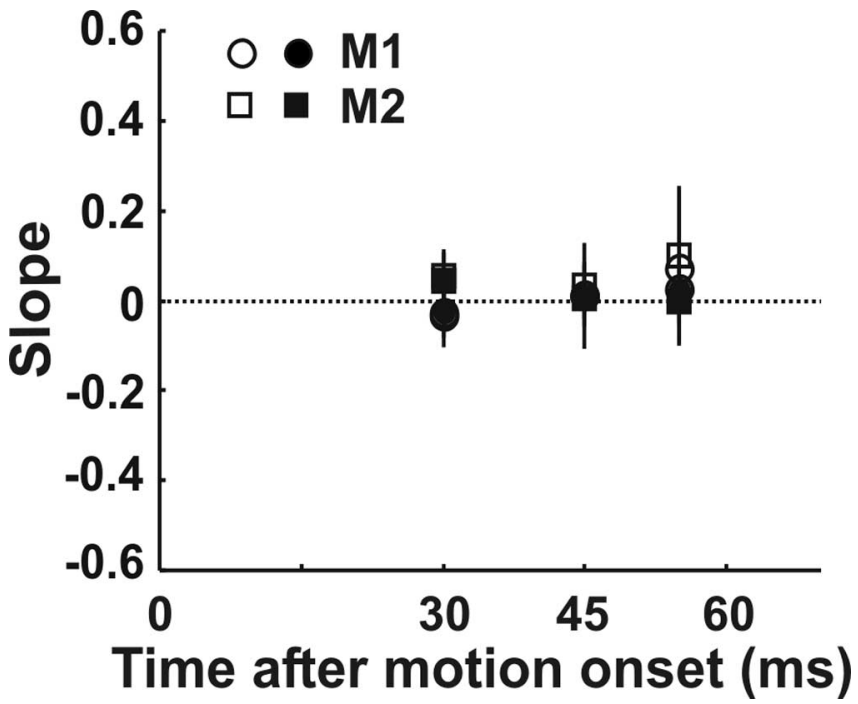

Figure 8. Fore-aft motion: test of the foveal and flexible goal visual strategy hypotheses. Shown are the mean \pm SD Flexl versus Fovl slopes averaged across all motion onset delays and measured at three different times after motion onset ( 30,45 , and $55 \mathrm{~ms})$. Data are plotted separately for the two animals, M1 (circles) and M2 (squares), as well as for the bar release (filled symbols) and saccade (open symbols) tasks. Dotted line is set at zero slope.

likely was allocated to the peripheral target just before the initiation of the saccade, yet even then responses were identical to those during steady fixation. Thus the intention to make a saccade to a peripheral location did not change the compensatory eye movement goal, not even a few milliseconds just before the saccade (Fig. 6). This result provides strong evidence for a hardwired invariant foveal goal. Nevertheless, we cannot exclude the possibility that high acuity-dependent attention (e.g., discrimination) tasks would reveal a flexible goal strategy, and such experiments have yet to be performed. Our data, however, clearly show that the foveal strategy is maintained at least for the simpler tasks used in the present experiments. As will be elucidated below, we propose that this compromise in functional flexibility arises because of two important needs: (1) processing speed for the generation of these eye movements and (2) efficiency in the extraction of heading information from retinal flow during self-motion.

Speed of vestibulo-motor responses

An important advantage for a hard-wired foveal strategy is a presumed simplification of the neural substrates and computations. As schematically illustrated by the flow patterns in Figure 1, the eye movements necessary for image stabilization must exhibit a complex dependence on both heading direction and the threedimensional spatial location (in terms of both horizontal/vertical eccentricities and depth) of the target of interest. If the spatial location to be stabilized is always the fovea, this information can be specified uniquely by the current position of the two eyes.

Consistent with this interpretation are results from previous studies showing that the scaling of the eye velocity amplitude by depth cues is limited to motor signals related to vergence angle and accommodation (Schwarz and Miles, 1991) and not disparity-related sensory information (Wei et al., 2003). By the exclusion of complex viewing distance computations from visual cues (Wei et al., 2003), potential spatial attention influences (this study), and limitation of TVOR scaling to efference copy information about binocular eye position, a fast and efficient visuomotor response is ensured. 


\section{Visual acuity and spatial navigation: two aspects of optic flow during self-motion?}

Processing speed and the simplification in the underlying neural correlates might not be the sole explanation for the absence of a more functionally relevant flexible goal visual strategy during self-motion. An additional functional advantage for a foveal visual strategy can be found in the use of retinal flow for spatial navigation. In particular, the retinal flow patterns that typically are experienced during self-motion [often referred to as optic flow (Gibson, 1950, 1954)] long have been known to be important for spatial navigation and heading perception (Berthoz et al., 1975; Warren and Hannon, 1988; Warren et al., 1988, 1991, 2001; Royden et al., 1994). However, optic flow is altered during eye movements. This property has been studied extensively during pursuit, in which either an extraretinal oculomotor signal or depth and motion parallax cues have been proposed as mechanisms to compensate for the shift in the focus of expansion when optic flow is used to judge heading (Warren and Hannon, 1990; Royden et al., 1992, 1994; van den Berg, 1993; Royden, 1994; van den Berg and Brenner, 1994; Wertheim, 1994; Banks et al., 1996).

In a similar way as pursuit, the reflexive eye movements described here would alter the experienced optic flow by shifting the focus of expansion away from the true heading direction. Thus it is essential that, under natural circumstances in which typically our eyes do not remain stationary during self-motion, the brain differentiates between a singularity in the visual field and actual heading by correcting for these compensatory eye movements. In the latter case, the heading can be computed directly from the equations relating compensatory eye velocity with the corresponding fixation position (Angelaki and Hess, 2005). Specifically, because for a foveal goal there is a fixed geometrical relationship between reflexive eye movements and motion direction, computation of heading parameters is simplified to solving simple algebraic equations (Angelaki and Hess, 2005). Thus, as long as these eye movements follow a fixed and invariant foveal goal, the task of disambiguating optic flow during self-motion represents an easy and tractable task. If, however, this relationship is broken down and compensatory eye velocity is dissociated from the corresponding eye position (as it would happen with the flexible goal hypothesis), computation of heading would become more complex. Thus we propose that the invariant foveal stabilization goal for the vestibularly driven eye movements during self-motion represents an effective compromise between the two potentially opposing effects of optic flow during self-motion. When maintenance visual acuity is limited to the fovea, the interpretation and use of optic flow for spatial navigation are greatly simplified.

\section{References}

Angelaki DE (1998) Three-dimensional organization of otolith-ocular reflexes in rhesus monkeys. III. Responses to translation. J Neurophysiol 80:680-695.

Angelaki DE (2002) Dynamics and viewing distance dependence of eye movements during transient lateral motion. Arch Ital Biol 140:315-322.

Angelaki DE, Hess BJM (2001) Direction of heading and vestibular control of binocular eye movements. Vision Res 41:3215-3228.

Angelaki DE, Hess BJM (2005) Self-motion-induced eye movements: effects on visual acuity and navigation. Nat Rev Neurosci 6:966-976.

Angelaki DE, McHenry MQ (1999) Short-latency primate vestibulo-ocular responses during translation. J Neurophysiol 82:1651-1654.

Banks MSH, Ehrlich SM, Backus BT, Crowell JA (1996) Estimating heading during real and simulated eye movements. Vision Res 36:431-443.

Bashinski HS, Bacharach VR (1980) Enhancement of perceptual sensitivity as the result of selectively attending to spatial locations. Percept Psychophys 28:241-248.
Berthoz A, Pavard B, Young LR (1975) Perception of linear horizontal selfmotion induced by peripheral vision (linearvection) basic characteristics and visual-vestibular interactions. Exp Brain Res 23:471-489.

Bowman EM, Brown VJ, Kertzman C, Schwarz U, Robinson DL (1993) Covert orienting of attention in macaques. I. Effects of behavioral context. J Neurophysiol 70:431-443.

Busettini C, Masson GS, Miles FA (1997) Radial optic flow induces vergence eye movements with ultra-short latencies. Nature 390:512-515.

Bush GA, Miles FA (1996) Short-latency compensatory eye movements associated with a brief period of free fall. Exp Brain Res 108:337-340.

Butter CM, Buchtel HA, Santucci R (1989) Spatial attentional shifts, further evidence for the role of polysensory mechanisms using visual and tactile stimuli. Neuropsychologia 27:1231-1240.

Carrasco M, Penpeci-Talgar C, Eckstein M (2000) Spatial covert attention increases contrast sensitivity across the CSF: support for signal enhancement. Vision Res 40:1203-1215.

Carrasco M, Ling S, Read S (2004) Attention alters appearance. Nat Neurosci 7:308-313.

Coleman TF, Li Y (1996) An interior trust region approach for nonlinear minimization subject to bounds. SIAM J Optim 6:418-445.

Cook EP, Maunsell JH (2004) Attentional modulation of motion integration of individual neurons in the middle temporal visual area. J Neurosci 24:7964-7977.

Downing CJ (1988) Expectancy and visual-spatial attention effects on perceptual quality. J Exp Psychol Hum Percept Perform 14:188-202.

Gibson JJ (1950) The perception of the visual world. Boston: Houghton Mifflin.

Gibson JJ (1954) The visual perception of objective motion and subjective movement. Psychol Rev 61:304-314.

Goldberg ME, Wurtz RH (1972) Activity of superior colliculus in behaving monkey. II. Effect of attention on neuronal responses. J Neurophysiol 35:560-574.

Hess BJM, Angelaki DE (2003) Vestibular contributions to gaze stability during transient forward and backward motion. J Neurophysiol 90:1996-2004.

Judge SJ, Richmond BJ, Chu FC (1980) Implantation of magnetic search coils for measurement of eye position, an improved method. Vision Res 20:535-538.

Kodaka Y, Wada Y, Kawano K (2003) Vergence responses to forward motion in monkeys: visual modulation at ultra-short latencies. Exp Brain Res 148:541-544.

Kowler E, Anderson E, Dosher B, Blaser E (1995) The role of attention in the programming of saccades. Vision Res 35:1897-1916.

Kustov AA, Robinson DL (1996) Shared neural control of attentional shifts and eye movements. Nature 384:74-77.

Li N, Wei M, Angelaki DE (2005) Primate memory saccade amplitude after intervened motion depends on target distance. J Neurophysiol 94:722-733.

McHenry MQ, Angelaki DE (2000) Primate translational vestibulo-ocular reflexes. II. Version and vergence responses to fore-aft motion. J Neurophysiol 83:1648-1661.

Moore T, Fallah M (2001) Control of eye movements and spatial attention. Proc Natl Acad Sci USA 98:1273-1276.

Paige GD, Tomko DL (1991) Eye movement responses to linear head motion in the squirrel monkey. I. Basic characteristics. J Neurophysiol 65:1170-1182.

Posner M (1980) Orienting of attention. Q J Exp Psychol 32:3-25.

Reynolds JH, Pasternak T, Desimone R (2000) Attention increases sensitivity of V4 neurons. Neuron 26:703-714.

Robinson DA (1963) A method of measuring eye movement using a scleral search coil in a magnetic field. IEEE Trans Biomed Eng 10:137-145.

Royden CS (1994) Analysis of misperceived observer motion during simulated eye rotations. Vision Res 34:3215-3222.

Royden CS, Banks MS, Crowell JA (1992) The perception of heading during eye movements. Nature 360:583-585.

Royden CS, Crowell JA, Banks MS (1994) Estimating heading during eye movements. Vision Res 34:3197-3214.

Savitsky A, Golay MJE (1964) Smoothing and differentiation of data by simplified least squares procedures. Anal Chem 36:1627-1639.

Schwarz U, Miles FA (1991) Ocular responses to translation and their dependence on viewing distance. I. Motion of the observer. J Neurophysiol $66: 851-864$. 
Schwarz U, Busettini C, Miles FA (1989) Ocular responses to linear motion are inversely proportional to viewing distance. Science 245:1394-1396.

van den Berg AV (1993) Perception of heading. Nature 365:497-498.

van den Berg AV, Brenner E (1994) Humans combine the optic flow with static depth cues for robust perception of heading. Vision Res 34:2153-2167.

Warren Jr WH, Hannon DJ (1988) Direction of self-motion is perceived from optical flow. Nature 336:162-163.

Warren Jr WH, Hannon DJ (1990) Eye movements and optical flow. J Opt Soc Am [A] 7:160-169.

Warren Jr WH, Morris MW, Kalish M (1988) Perception of translational heading from optical flow. J Exp Psychol Hum Percept Perform 14:646-660.

Warren Jr WH, Mestre DR, Blackwell AW, Morris MW (1991) Perception of circular heading from optical flow. J Exp Psychol Hum Percept Perform 17:28-43.
Warren Jr WH, Kay BA, Zosh WD, Duchon AP, Sahuc S (2001) Optic flow is used to control human walking. Nat Neurosci 4:213-216.

Wei M, Angelaki DE (2004) Viewing distance dependence of the vestibuloocular reflex during translation: extra-otolith influences. Vision Res 44:933-942.

Wei M, DeAngelis GC, Angelaki DE (2003) Do visual cues contribute to the neural estimate of viewing distance used by the oculomotor system? J Neurosci 23:8340-8350.

Wertheim AH (1994) Motion perception during self-motion: the direct versus inferential controversy revisited. Behav Brain Sci 17:293-355.

Wurtz RH, Mohler CW (1976) Organization of monkey superior colliculus: enhanced visual response of superficial layer cells. J Neurophysiol 39:745-765.

Yang DS, Fitzgibbon EJ, Miles FA (1999) Short-latency vergence eye movements induced by radial optic flow in humans: dependence on ambient vergence level. J Neurophysiol 81:945-949. 